\title{
Mixed Finite Elements for Variational Surface Modeling
}

\author{
Alec Jacobson ${ }^{1}$, Elif Tosun ${ }^{1,2}$, Olga Sorkine ${ }^{1}$ and Denis Zorin $^{1}$ \\ ${ }^{1}$ New York University, USA $\quad{ }^{2}$ Solidworks, USA
}

\begin{abstract}
Many problems in geometric modeling can be described using variational formulations that define the smoothness of the shape and its behavior w.r.t. the posed modeling constraints. For example, high-quality $C^{2}$ surfaces that obey boundary conditions on positions, tangents and curvatures can be conveniently defined as solutions of high-order geometric PDEs; the advantage of such a formulation is its conceptual representation-independence. In practice, solving high-order problems efficiently and accurately for surfaces approximated by meshes is notoriously difficult. For modeling applications, the preferred approach is to use discrete geometric schemes which are efficient and robust, but their convergence properties are less well understood compared to higher-order FEM. In this paper, we explore discretizations of common geometric PDEs on meshes using mixed finite elements, where additional variables for the derivatives in the problem are introduced. Such formulations use first-order derivatives only, allowing a discretization with simple linear elements. Various boundary conditions can be naturally discretized in this setting. We formalize continuous region constraints commonly used in modeling applications, and show that these seamlessly fit into the mixed framework. We demonstrate that some of the commonly used discrete geometric discretizations can be regarded as a particular case of mixed finite elements. We study the convergence behavior of our discretizations, and how they can be applied to implement common modeling tasks.
\end{abstract}

Categories and Subject Descriptors (according to ACM CCS): I.3.5 [Computer Graphics]: Computational Geometry and Object Modeling-Geometric algorithms, languages, and systems

\section{Introduction}

A variety of geometric modeling problems are solved using energy minimization or geometric PDEs: defining a smooth surface interpolating fixed points or curves, filling holes and connecting pieces of geometry, deformations, and cut-andpaste operations. Such energies and PDEs are defined in terms of differential quantities such as tangents, curvatures and curvature derivatives. Boundary conditions for these problems play an important role, for instance ensuring that surfaces are joined smoothly. Higher-order boundary conditions can only be imposed if the variational problems are of high order: $C^{1}$ and $C^{2}$ conditions can be imposed for PDEs of order at least four and six, respectively.

While the PDEs of interest have high order, they usually need to be solved for surfaces approximated by meshes, and numerical schemes that produce mesh-independent solutions in the limit of fine meshes and remain efficient need to be devised. Many reliable finite element discretizations are available for fourth-order problems, yet all standard conforming and non-conforming elements require additional degrees of freedom associated with derivatives at vertices and edge midpoints, increasing the overall number of variables and complicating implementation. Furthermore, sixth-order problems, which are highly important in geometric modeling applications, rarely appear in finite-element literature.

A currently preferred alternative in many geometry processing applications is to formulate discrete analogs of variational problems using discrete-geometric operators, such as discrete Laplacians. These approaches are simple, robust and perform well in practice, but lack the mesh-independence guarantees provided by the finite elements designed to be convergent, although mesh-independent and convergent behavior was observed experimentally for biharmonic problems on a range of mesh types [GGRZ06]. An additional important complication is a systematic treatment of boundary conditions: while satisfactory heuristics were designed for a number of cases (most importantly, for region conditions, see Section 3), other types of conditions, such as direct specification of tangents or curvature along a boundary, are more difficult and typically require ad hoc mesh extension.

In this paper, we present a discretization for two common PDEs on meshes using mixed finite elements, a wellestablished finite-element technique. The main idea is to introduce additional variables for the derivatives in the prob- 
lem, along with additional constraint equations relating these variables to the original ones, thereby reducing a high-order equation to a low-order system which can be discretized with linear elements. We show that the intuitive and commonly used discretization of biharmonic and triharmonic equations introduced in [BK04] can be viewed as a transformation of a mixed element discretization, and is directly related to the Ciarlet-Raviart discretization of the biharmonic equation. For the triharmonic equation, we show that a standard mixed FEM discretization may lead to a singular system, but an alternative formulation with region constraints does not suffer from this problem. We show how different types of boundary conditions commonly used for geometric problems can be discretized with mixed elements. We formalize the notion of continuous region constraints, which are common in geometric modeling but rarely considered in finite element literature, and demonstrate that these also naturally fit into the mixed framework.

We explore the convergence behavior of mixed discretizations for a variety of mesh types and evaluate the degree of mesh dependence of the discretization. We demonstrate several applications of the mixed framework in geometric modeling, such as interactive shape editing, hole filling, blending, and surface patch construction from boundary curves.

\section{Previous work}

Many works in graphics and geometric modeling have considered surface design based on PDEs. In the following we briefly describe the most relevant literature, classifying it by the type of PDEs addressed, the employed discretization and the possible boundary conditions.

In the special case of simple domains and analytically specified boundary conditions, PDEs can be solved in closed form without any discretization [BW90]. In more general settings, the equations need to be discretized and solved numerically. Moreton and Séquin [MS92] model shapes that minimize the curvature variation energy, where positions, tangents and normal curvatures are specified along curves, and Bézier patches are used to approximate the surface. Welch and Witkin [WW94] also use curvature variation to interpolate curve networks and points, but on general triangular meshes; they compute the required differential quantities with local quadratic fits and finite differences.

Local quadratic fits may suffer from instabilities, unless sufficiently large number of vertices are used, which results in lower performance. To gain speed and accuracy (by using much finer meshes) many recent approaches adopt discrete differential operators such as the discrete Laplacians. Taubin [Tau95] used the uniform-weight discrete Laplacian for fair surface design and proposed constraining point positions or discrete Laplacian values at vertices. Later works use the more accurate discrete cotangent Laplacian [PP93], closely related to $\mathrm{FE}$ discretization [ $\left.\mathrm{WBH}^{*} 07, \mathrm{RBG}^{*} 09\right]$ ]

Mixed finite elements are based on factoring a higherorder problem into a system of lower-order problems. This idea is also used in the context of discrete differential operators when boundary conditions allow this, e.g. Schneider and Kobbelt [SK00, SK01] factor the PDE $\Delta H=0$ into two second-order equations. In the FIBERMESH system [NISA07], the same approach is used to construct fair surfaces that interpolate a set of arbitrary curves, with no tangents or curvatures fixed. A more complete survey of discrete variational techniques can be found in [BS08]. A priori, these approaches lack convergence guarantees for general meshes. In absence of convergence, the degree of mesh dependence is hard to predict, and adaptive refinement techniques are hard to apply. In [GGRZ06], it is demonstrated that the discretization of the Laplacian energy (equivalently, the biharmonic equation) based on the cotangent weights does not satisfy a version of the patch test (a standard test used for verifying convergence of finite elements in engineering) yet empirically it exhibits convergent behavior. Mixed-element point of view described in this paper provides a different approach for analysis of this discretization.

The closest work to ours is the method of Clarenz et al. [CDD*04] that applies the Willmore flow (fourth-order geometric PDE) to fair surface design by using FEM with auxiliary variables $\mathbf{y}=\Delta \mathbf{x}$. Their formulation allows prescribing $\mathbf{x}$ values and co-normals on the boundary. We demonstrate that this and other discrete approaches can be viewed as an application of the mixed finite element discretization, and that a variety of discrete boundary conditions (region conditions) used in [BK04], [CDD*04] and [XPB06] can be derived from a continuous formulation.

Finite elements offer a consistent way of solving geometric PDEs and treating various boundary conditions; however, relatively complex higher-order elements are required for conforming discretization of higher-order PDEs. Nonconforming elements such as DKT, are widely used in engineering for fourth-order problems but are difficult to extend to higher orders and require many additional derivativerelated degrees of freedom. Some of the early work applied standard engineering elements to surface modeling [CG91], yet most methods, especially for interactive applications, used simpler discretizations for reasons of efficiency and implementation ease. Mixed finite elements for fourth-order problems offer an alternative approach that allows using piecewise linear basis functions. Although additional variables are introduced, they have a natural interpretation and in most cases can be eliminated inexpensively. A mixed formulation was introduced for the biharmonic equation in [CR74] and its variations are considered in [Fal78, Mon87, AD01] and many other papers. Convergence of mixed discretization for linear elements was shown in [Sch78]. In contrast to biharmonic equations, sixth-order equations (especially triharmonic) did not receive much attention, as few physical problems result in such equations. One exception is [BF85], deriving theoretical convergence estimates for a mixed formulation of polyharmonic equations. While piecewise-linear discretizations extend naturally to the case of non-flat domains, convergence questions require special treatment; the Laplace-Beltrami equation and Willmore flow discretizations were analyzed in [Dzi88, Dzi90, DD06].

\section{Model problems}

We consider two important examples: Laplacian and Laplacian gradient energies, leading to biharmonic and trihar- 


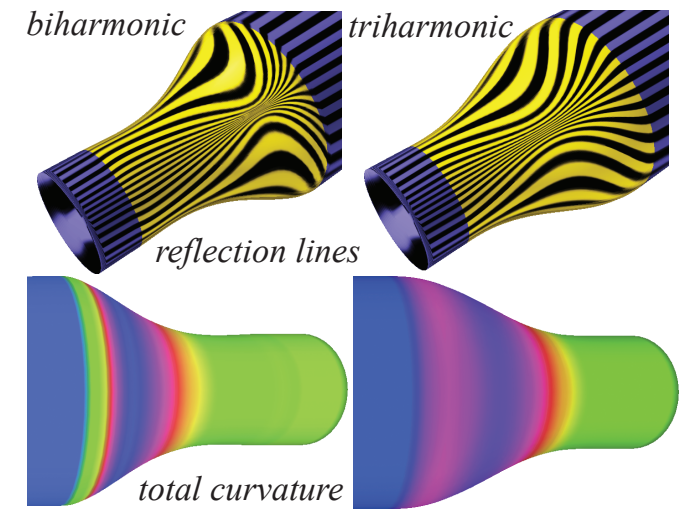

Figure 1: Blending two cylinders of different diameters using triharmonic and biharmonic equations.

monic equations (Fig. 1). We use the notation $\langle f, g\rangle_{X}=$ $\int_{X} f g d A$ for the $L^{2}$ inner product of two functions on a domain $X$ (an area or a curve in the plane or on a surface). When the subscript is omitted, the domain $\Omega$ is implied. For vector-valued functions, denoted by bold letters, the product in the integral is replaced by dot product.

In the first problem we compute a deformation $\mathbf{u}$ of a planar sheet occupying an area $\Omega_{0}$ in the plane so that the Laplacian energy is minimized:

$$
E_{B}=\frac{1}{2}\langle\Delta \mathbf{u}, \Delta \mathbf{u}\rangle_{\Omega_{0}} \rightarrow \min ,
$$

where $\mathbf{u}: \Omega_{0} \rightarrow \mathbb{R}^{3}$ is the deformation. The related EulerLagrange equation is the biharmonic equation $\Delta^{2} \mathbf{u}=0$.

The second example is the Laplacian gradient energy

$$
E_{T}=\frac{1}{2}\langle\nabla \Delta \mathbf{u}, \nabla \Delta \mathbf{u}\rangle_{\Omega_{0}} \rightarrow \min
$$

with boundary conditions on second derivatives, important for modeling curvature-continuous surfaces. The EulerLagrange equation for this problem is sixth order: $\Delta^{3} \mathbf{u}=0$.

Low-order decomposition. A general systematic approach to discretization of problems involving high-order derivatives is to convert an unconstrained optimization problem like (1) into a lower-order constrained optimization problem with additional variables. Instead of the Euler-Lagrange equations for (1), we solve the constrained problem

$$
\frac{1}{2}\langle\mathbf{v}, \mathbf{v}\rangle_{\Omega_{0}} \rightarrow \min , \text { s.t. } \Delta \mathbf{u}=\mathbf{v} .
$$

Similarly, instead of minimizing energy $E_{T}$, we solve

$$
\frac{1}{2}\langle\nabla \mathbf{v}, \nabla \mathbf{v}\rangle_{\Omega_{0}} \rightarrow \min , \text { s.t. } \Delta \mathbf{u}=\mathbf{v} .
$$

For the Laplacian gradient energy, we no longer need to discretize third derivatives. Furthermore, in both cases one can also eliminate second derivatives by using Green's identity:

$$
\begin{array}{r}
L_{B}=\frac{1}{2}\langle\mathbf{v}, \mathbf{v}\rangle_{\Omega_{0}}+\langle\lambda, \Delta \mathbf{u}-\mathbf{v}\rangle_{\Omega_{0}}= \\
\frac{1}{2}\langle\mathbf{v}, \mathbf{v}\rangle_{\Omega_{0}}-\langle\lambda, \mathbf{v}\rangle_{\Omega_{0}}+\left\langle\lambda, \frac{\partial \mathbf{u}}{\partial n}\right\rangle_{\partial \Omega_{0}}-\langle\nabla \lambda, \nabla \mathbf{u}\rangle_{\Omega_{0}}
\end{array}
$$

The partial derivative $\partial / \partial n$ is w.r.t. the normal of the boundary curve. A similar transformation yields a formulation with first-order derivatives for the minimization of $E_{T}$. Similar considerations apply for weak formulations of high-order PDEs in general. For the transformed problems, piecewiselinear elements can be used for all quantities, as it is commonly done for second-order problems. This idea is the foundation of the mixed finite element discretizations (Section 4). The constraint-based formulation has an additional significant advantage: it allows us to treat region boundary conditions in a systematic way, as explained below.

Boundary condition types. We define various boundary conditions in the continuous case. While for some applications (e.g., deformations of a fixed mesh) the problem can be studied entirely in the discrete domain, we are concerned with cases when a common reference point is needed for meshes with different connectivity and resolution that approximate the same shape.

A boundary condition can be associated with subsets of the domain $\Omega_{0}$ of different dimensions:

- region boundary conditions on open domains $\Omega_{f} \subset \Omega_{0}$;

- curve boundary conditions on curves $C \subset \Omega_{0}$;

- point boundary conditions on isolated points $P \in \Omega_{0}$.

Fixed region boundary conditions require that, given a domain $\Omega_{f}$ and a fixed function $\mathbf{u}_{f}$,

$$
\left.\mathbf{u}\right|_{\Omega_{f}}=\mathbf{u}_{f} \text {, and } \mathbf{u} \text { is } C^{k} \text { on } \Omega \text {, for some } k \leq 2 .
$$

These boundary conditions are illustrated in Fig. 2(left). This type of conditions can be viewed as the continous analog of conditions defined directly for meshes in [CDD*04,XPB06].

Fixed curve boundary conditions are most commonly considered in FE setting. On a curve $C$ (typically a part of $\partial \Omega$, but possibly in the interior of $\Omega_{0}$ ), we require that

$$
\left.\frac{\partial^{i} \mathbf{u}}{\partial n^{i}}\right|_{C}=\mathbf{b}_{i}, \quad i \leq k \text { for } k \leq 2 .
$$

Fig. 3(left) demonstrates the usage of this condition for explicit tangent control on a biharmonic surface. Triharmonic surface allows to specify both tangents and curvature (second derivative) at the boundary, as shown in Fig. 3(right).

Free (natural) curve boundary conditions are naturally obtained from energy-based problems; they are needed at a boundary with no Dirichlet constraints in order for the energy to be minimal. They can also be applied to PDEs not originating from an energy, yielding similar boundary behavior. These conditions have more complex explicit form involving high-order quantities, but no special effort is needed to enforce them when the discretization is derived from the energy, hence we do not consider them in detail.

Point boundary conditions are identical to region boundary conditions, except instead of a function defined on $\Omega_{f}$, we use a set of values defined at isolated points $\mathbf{p}_{i}$ of $\Omega_{0}$. An example is shown in Fig. 2(right).

\section{Mixed finite element discretization}

In this section, we demonstrate, using two model examples discussed in Section 3, how low-order decomposition can be used to discretize such problems with piecewise-linear elements only, and how different types of boundary conditions can be imposed. 


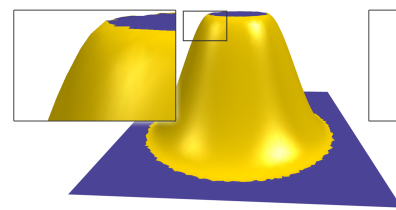

Region condition: $\Delta^{2} \mathbf{u}=0$

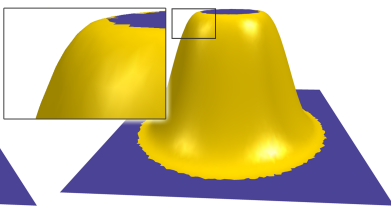

$\Delta^{3} \mathbf{u}=0$

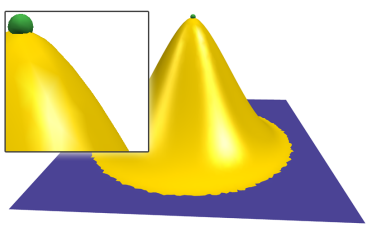

Point condition: $\Delta^{2} \mathbf{u}=0$

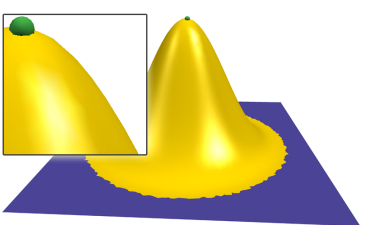

$\Delta^{3} \mathbf{u}=0$

Figure 2: Region and point boundary conditions. Left: the blue areas $\left(\Omega_{f}\right)$ are fixed and the yellow part $(\Omega)$ is solved for. The circular region in the middle was lifted upwards. Solving $\Delta^{k} \mathbf{u}=0$ with region boundary conditions. Right: the point constraint is highlighted by the green dot. In both examples, triharmonic surface is smoother around the boundary $\left(C^{2}\right)$.

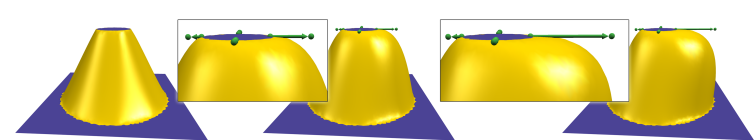

prescribing tangents on boundary curve, $\Delta^{2} \mathbf{u}=0$

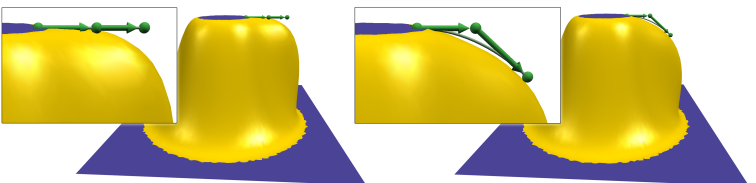

prescribing second derivatives perp. to boundary, $\Delta^{3} \mathbf{u}=0$

Figure 3: Prescribing curve boundary conditions. The leftmost image shows a biharmonic surface with $\partial \mathbf{u} / \partial n=0$. Tangents can be explicitly manipulated by the user (see also Fig. 4). The two rightmost images show a triharmonic surface with boundary curvature manipulation via a Bézier control widget.

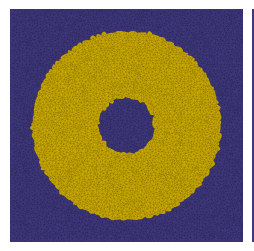

meshed domain

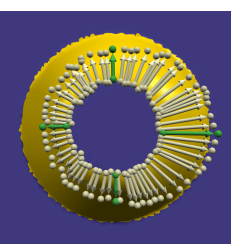

tangent constraints (see Fig. 3)

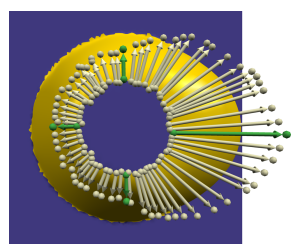

Figure 4: The mesh domain used in our examples for region and curve boundary conditions. Tangent vectors can be explicitly manipulated by the user (see the green vectors in the right two images); the rest of the tangents along the boundary are automatically interpolated (shown in light brown).

Our main focus is on the region boundary conditions which lack a systematic treatment in finite element context. We demonstrate the relationship between the classical Ciarlet-Raviart mixed-element discretization of the biharmonic equation [CR74], discrete-geometric discretization of [BK04] and our region constraint formulation.

Notation. Please refer to Fig. 5. We assume that the domain $\Omega_{0}$ has polygonal boundary and is meshed (the discretizations we describe, with some restrictions, are applicable to domains with curved boundaries approximated by polygonal domains $\Omega_{0}^{h}$ for each resolution). The parameter $h$ denotes the average edge length of the mesh. The domain $\Omega$ is defined as the subset of $\Omega_{0}$ excluding the union of $\Omega_{f}$ with constrained curves and points, and the complement of $\Omega$ in $\Omega_{0}$ is denoted by $\Omega_{e}$.

The set of vertex indices in the interior of $\Omega$ is denoted by $I_{\Omega}$, the set of vertex indices on the boundary of $\Omega$ is $I_{0}$, the sets of indices in $\Omega_{e}$ in two layers outside the boundary are $I_{1}$ and $I_{2}$. The set of all vertex indices in $\Omega_{0}$ is denoted by $I$, and the set of vertices in $\Omega_{e}$ is denoted by $I_{e}$. In a matrix $S$ with rows and columns corresponding to vertex indices, we use subscripts $\Omega, 0,12$ and $e$, to define submatrices with

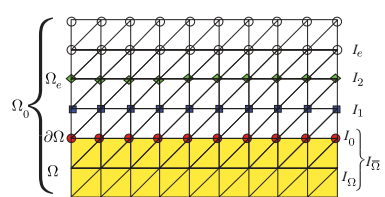

Figure 5: Notation for different areas of the mesh; for fourth-order equations, we include $I_{2}$ into $I_{e}$.

rows and columns coming from corresponding index sets. We also use subscripts like 01 to denote $I_{1} \cup I_{0}$, and $\bar{\Omega}$ for $I_{\Omega} \cup I_{0}$, i.e., $\Omega$ with its boundary included.

\subsection{Laplacian energy $\&$ biharmonic equation}

We start with the very well studied example of Laplacian energy (1), whose Euler-Lagrange equation is the biharmonic equation. It reveals the essential relations between conventional mixed finite element discretizations, the region constraint discretization that we propose, and the discretegeometric approach of [BK04].

Region conditions require an additional term in the Lagrangian, constraining the solution to coincide with a given function on $\Omega_{f}$ :

$$
\begin{aligned}
& \frac{1}{2}\langle\mathbf{v}, \mathbf{v}\rangle_{\Omega_{0}}-\langle\lambda, \mathbf{v}\rangle_{\Omega_{0}}+\left\langle\lambda, \frac{\partial \mathbf{u}}{\partial n}\right\rangle_{\partial \Omega}-\langle\nabla \lambda, \nabla \mathbf{u}\rangle_{\Omega_{0}} \\
& +\left\langle\mu, \mathbf{u}_{f}-\mathbf{u}\right\rangle_{\Omega_{f}}
\end{aligned}
$$

where $\mu$ is the Lagrange multiplier function for the region constraint. It is important to note that the first constraint, $\Delta \mathbf{u}=\mathbf{v}$, is enforced over all of $\Omega_{0}$, which ensures $C^{1}$ continuity across the boundary of $\Omega$.

A similar Lagrangian, with the first term replaced with $\langle\nabla \mathbf{v}, \nabla \mathbf{v}\rangle_{\Omega_{0}}$ is obtained for the problem (2):

$$
\begin{aligned}
& \frac{1}{2}\langle\nabla \mathbf{v}, \nabla \mathbf{v}\rangle_{\Omega_{0}}-\langle\lambda, \mathbf{v}\rangle_{\Omega_{0}}+\left\langle\lambda, \frac{\partial \mathbf{u}}{\partial n}\right\rangle_{\partial \Omega}-\langle\nabla \lambda, \nabla \mathbf{u}\rangle_{\Omega_{0}} \\
& +\left\langle\mu, \mathbf{u}_{f}-\mathbf{u}\right\rangle_{\Omega_{f}}
\end{aligned}
$$


Discretization with piecewise linear elements. We use piecewise-linear approximations for $\mathbf{u}, \mathbf{v}, \lambda$ and $\mu$, all of the form $\sum_{i \in I} a_{i} \varphi_{i}$. For a quantity $a$, we denote the piecewise linear approximation by $a^{h}$.

Substituting these approximations into the Lagrangian, and differentiating w.r.t. all free variables $\mathbf{v}_{i}, \mathbf{u}_{i}, \lambda_{i}, \mu_{i}$, defined at vertices $i \in I \backslash I_{\partial \Omega_{0}}$, we obtain the following system of equations:

$$
\begin{aligned}
\sum_{j \in I}\left(\mathbf{v}_{j}-\lambda_{j}\right)\left\langle\varphi_{i}, \varphi_{j}\right\rangle & =0 \\
-\sum_{j \in I} \lambda_{j}\left\langle\nabla \varphi_{i}, \nabla \varphi_{j}\right\rangle-\sum_{j \in I} \mu_{j}\left\langle\varphi_{j}, \varphi_{i}\right\rangle_{\Omega_{f}} & =0 \\
-\sum_{j \in I} \mathbf{u}_{j \in I}\left\langle\nabla \varphi_{i}, \nabla \varphi_{j}\right\rangle+\sum_{j} \mathbf{v}_{j}\left\langle\varphi_{j}, \varphi_{i}\right\rangle & =0 \\
\sum_{j \in I}\left(\mathbf{u}_{j}-\mathbf{u}_{j}^{f}\right)\left\langle\varphi_{j}, \varphi_{i}\right\rangle_{\Omega_{f}} & =0
\end{aligned}
$$

The first equation allows us to eliminate $\lambda_{i}$ immediately as it has to coincide with $\mathbf{v}_{i}$, leaving variables $\mathbf{u}, \mathbf{v}$ and $\mu$. One can observe that the coefficients of the system mostly come from the discrete Laplacian matrix $L_{i j}=-\left\langle\nabla \varphi_{i}, \nabla \varphi_{j}\right\rangle$, and the mass matrix $M_{i j}^{\text {full }}=\left\langle\varphi_{i}, \varphi_{j}\right\rangle$.

Lumped mass matrices. The mass matrix $M^{\text {full }}$ is often replaced by a lumped mass matrix, i.e. a diagonal matrix $M^{d}$. The lumping step is mathematically sound: replacing $\left\langle\mathbf{v}^{h}, \mathbf{v}^{h}\right\rangle=\mathbf{v}^{h^{T}} M \mathbf{v}^{h}$ with $\mathbf{v}^{h^{T}} M^{d} \mathbf{v}^{h}$ does not affect the convergence rate for the solution, if the quadrature rule has accuracy $O(h)$, which is satisfied by using vertices as quadrature points [Cia78, BF91], which leads to a diagonal mass matrix. From now on, we assume that the mass matrix in (8) was replaced by a diagonal matrix $M^{d}$ whose diagonal entries are denoted by $D_{i}$; two approaches to computing $D_{i}$ are briefly compared in Section 5. With a diagonal mass matrix, the system reduces to

$$
\begin{array}{ll}
\sum_{j \in I} \mathbf{v}_{j} L_{i j}-D_{i} \mu_{i}=0 \text { if } i \in I_{e}, & \sum_{j \in I} \mathbf{v}_{j} L_{i j}=0 \text { if } i \notin I_{e} ; \\
\sum_{j \in I} L_{i j} \mathbf{u}_{j}-D_{i} \mathbf{v}_{i}=0 ; & \sum_{j \in I} \mathbf{u}_{j}-\mathbf{u}_{j}^{f}=0 \text { if } i \in I_{e} .
\end{array}
$$

As the values of the Lagrange multiplier $\mu$ are not of interest to us, we can eliminate the first set of equations defining $\mu_{i}$ in terms of $\mathbf{v}_{i}$, since $\mu_{i}$ are not present in other equations. The last set of equations indicates that for $i$ on $I_{f}, \mathbf{u}_{i}$ can be replaced by the known values $\mathbf{u}_{i}^{f}$. Finally, we can compute the values of $\mathbf{v}_{i}$ for $i \in I_{e}$ using $\sum_{j \in I} L_{i j} \mathbf{u}_{j}=D_{i} \mathbf{v}_{i}$, as for entries away from the boundary, all $\mathbf{u}_{j}$ with nonzero $L_{i j}$ are fixed values $\mathbf{u}_{j}^{f}$.

Eliminating values of $\mathbf{u}_{j}$ and $\mathbf{v}_{j}$ in fixed regions from the system after swapping equations and moving known quantities to the right-hand side, yields in matrix form:

$$
\left[\begin{array}{cc}
-M^{d} & L_{\bar{\Omega}, \Omega} \\
L_{\Omega, \bar{\Omega}} & 0
\end{array}\right]\left[\begin{array}{l}
\mathbf{v}_{\bar{\Omega}} \\
\mathbf{u}_{\Omega}
\end{array}\right]=\left[\begin{array}{c}
-L_{\bar{\Omega}, 0} \mathbf{u}_{0}^{f}-L_{\bar{\Omega}, 1} \mathbf{u}_{1}^{f} \\
0
\end{array}\right] .
$$

The system can be simplified even further, if we observe that $M^{d}$ can be easily inverted, and $\mathbf{v}_{\bar{\Omega}}$ can be eliminated from the system, yielding

$$
L_{\Omega, \bar{\Omega}}\left(M^{d}\right)^{-1} L_{\bar{\Omega}, \Omega} \mathbf{u}_{\Omega}=-L_{\Omega, \bar{\Omega}}\left(M^{d}\right)^{-1} L_{\bar{\Omega}, 01} \mathbf{u}_{01}^{f},
$$

leaving only $\mathbf{u}_{\Omega}$ as the unknown.

Observe that point conditions are enforced using the same system of equations: for an isolated point constraint, assuming it is at a mesh vertex, $I_{1}$ is empty, so the second term of the right-hand side is absent.

Fixed curve conditions. Fixed-curve boundary conditions of the form $\mathbf{u}=\mathbf{b}_{0}$ and $\partial \mathbf{u} / \partial n=\mathbf{b}_{1}$ are commonly used in mixed discretizations of the biharmonic equation; we refer to [CR74, AD01] for details. An approach similar to the one outlined above can be used, with the Lagrangian in (5) restricted to $\bar{\Omega}$. This yields the system of equations

$$
\left[\begin{array}{cc}
-M^{\Omega} & L_{\bar{\Omega}, \Omega} \\
L_{\Omega, \bar{\Omega}} & 0
\end{array}\right]\left[\begin{array}{c}
\mathbf{v}_{\bar{\Omega}} \\
\mathbf{u}_{\Omega}
\end{array}\right]=\left[\begin{array}{c}
-L_{\bar{\Omega}, 0}^{\Omega} \mathbf{b}_{0}-N_{\bar{\Omega}, 0}^{\partial \Omega} \mathbf{b}_{1} \\
0
\end{array}\right],
$$

where the matrix $N_{i j}^{\partial \Omega}=\left\langle\varphi_{j}, \varphi_{i}\right\rangle_{\partial \Omega}$, and $L^{\Omega}$ and $M^{\Omega}$ are the discrete Laplacian and mass matrices with integration performed over $\Omega$ only, as $\langle\cdot\rangle_{\Omega}=\langle\cdot\rangle_{\Omega_{0}}-\langle\cdot\rangle_{\Omega_{e}}, L^{\Omega}=L-$ $L^{\Omega_{e}}$. This is exactly the Ciarlet-Raviart discretization for piecewise-linear elements with convergence established in [Sch78].

Comparing (12) and (10), we observe two differences: first, the right-hand side term $L_{\bar{\Omega}, 1} \mathbf{u}_{1}^{f}$ is replaced with $-L_{\bar{\Omega}, 1}^{\Omega_{e}} \mathbf{u}_{1}^{f}+N_{\bar{\Omega}, 0}^{\partial \Omega} \mathbf{b}_{1}$; second, the full mass matrix is retained (in this case, lumped matrices are not required to eliminate variables). A more detailed analysis presented in the appendix shows that the difference between the solutions of the two systems converges to zero as $h \rightarrow 0$. Replacing $M^{\Omega}$ with diagonal $M^{\Omega, d}$ yields a system matrix similar to (10), except the integration is over $\Omega$ only; the same elimination procedure can be applied to obtain a system for $\mathbf{u}$ alone.

Our observations are summarized in Proposition 1:

Proposition 1 The systems (10) and (11) are equivalent to the discretization presented in [BK04], and, up to a perturbation of the right-hand side and restricting mass-matrix integration to $\Omega$, to the Ciarlet-Raviart system (12).

We note that adjusting $\mathbf{b}_{1}$ offers a degree of control over how smoothly the surface approaches the boundary, similar to the control offered by the $\lambda$ parameter in [BK04] but without modifying the system matrix (which leads to more efficient computations) and with more direct interpretation.

Convergence. As we discuss in more detail in the appendix, this connection between (10) and (12) can be used to apply available theory for (10) and establish convergence. Note that the cotangent formula discretization of the Laplacian energy discussed in [GGRZ06] exactly matches (10). While it fails the consistency part of the version of the patch test discussed there, this, by itself, does not preclude convergence, which in the case of (10) is established by reduction to Ciarlet-Raviart system.

We emphasize that using a conforming FE discretization in the case of constrained problems like (10) does not 
guarantee convergence. In fact, the more general technique for analysis of mixed elements based on the LBB condition [BF91] cannot be applied to any discretization of the biharmonic equation. All known estimates are suboptimal, in a sense that the rate of convergence of the solution is lower than $O\left(h^{2}\right)$ approxmiation power of p.w. linear finite elements. The $H^{1}$ norm estimates in [Sch78] and [AD01], combined with Poincare-Friedrichs inequality lead to $L^{2}$ norm convergence rates $h^{7 / 4}(\log h)^{3 / 2}$.

\subsection{Laplacian gradient energy \& triharmonic equation}

The similarity between the Lagrangians (5) and (7) allows deriving a piecewise-linear discretization of the triharmonic system in a similar way: the main steps remain the same, namely $(i)$ use p.w. linear approximations for all functions; (ii) replace mass matrices with lumped mass matrices; (iii) eliminate unneeded variables. An important distinction is that the Lagrange multiplier $\lambda$ can no longer be eliminated, due to a more complex form of the equation obtained by differentiating with respect to $\mathbf{v}_{i}$. From direct examinaton of the system one observes that the Lagrange multiplier $\lambda$ corresponds to $\mathbf{w}$ in the low-order factorization of the triharmonic equation of the form $\Delta \mathbf{u}=\mathbf{v}, \Delta \mathbf{v}=\mathbf{w}, \Delta \mathbf{w}=0$.

Because of the different form of the first equation, we no longer can eliminate all variables corresponding to vertices outside the one-row neighborhood of $\bar{\Omega}$, and two rows are needed, as expected from the discrete-geometric discretizations of triharmonic equations. We obtain the following system in the end:

$$
\left[\begin{array}{ccc}
L_{\bar{\Omega} \bar{\Omega}} & 0 & -M_{\bar{\Omega} \bar{\Omega}}^{d} \\
0 & 0 & L_{\Omega \bar{\Omega}} \\
-M_{\bar{\Omega} \bar{\Omega}}^{d} & L_{\bar{\Omega} \Omega} & 0
\end{array}\right]\left[\begin{array}{c}
\mathbf{v}_{\bar{\Omega}} \\
\mathbf{u}_{\Omega} \\
\lambda_{\bar{\Omega}}
\end{array}\right]=\left[\begin{array}{c}
-L_{\bar{\Omega}, 1} \mathbf{v}_{1} \\
0 \\
-L_{\bar{\Omega}, 0} \mathbf{u}_{0}^{f}-L_{\bar{\Omega}, 1} \mathbf{u}_{1}^{f}
\end{array}\right]
$$

where

$$
\mathbf{v}_{1}=-L_{1,0} \mathbf{u}_{0}^{f}-L_{1,1} \mathbf{u}_{1}^{f}-L_{1,2} \mathbf{u}_{2}^{f} .
$$

Again, as it was observed for the biharmonic equation, one can also eliminate $\mathbf{v}$ and $\lambda$ entirely by inverting the mass matrices, and obtaining a system in terms of $\mathbf{u}$ alone, which coincides with the system of [BK04].

Curve boundary conditions. For curve boundary conditions, the discretization can be obtained in a similar way, however an important difference arises. In this case, the values $\mathbf{v}_{i}$ are fixed for boundary vertices, unlike in the case of the region boundary condition system (13), where these remain as free variables. This results in the system matrix

$$
\left[\begin{array}{ccc}
L_{\Omega \Omega} & 0 & -M_{\Omega \bar{\Omega}}^{\Omega, d} \\
0 & 0 & L_{\Omega \bar{\Omega}} \\
-M_{\bar{\Omega} \Omega}^{\Omega, d} & L_{\bar{\Omega} \Omega} & 0
\end{array}\right]
$$

Somewhat surprisingly, although the discretization follows the same pattern as well-established discretizations for biharmonic equations, the resulting systems are often overdetermined and cannot be practically used. The following proposition describes simple local mesh configurations leading to singularity, but one can observe more complex global dependencies, resulting from the fact that there are "too many" degrees of freedom fixed on the boundary, while the equations corresponding to boundary vertices are retained.

Proposition 2 The system (15) is singular if two vertices in $I_{1}$ have exactly one edge-adjacent vertex in $I_{\Omega}$.

Indeed, observe that the system with lumped mass contains the equations of the form $L_{52} \mathbf{u}_{2}=D_{5} \mathbf{v}_{5}-L_{54} \mathbf{u}_{4}-$ $L_{55} \mathbf{u}_{5}-L_{56} \mathbf{u}_{6}-L_{53} \mathbf{u}_{3}$, and $L_{52} \mathbf{u}_{2}=D_{3} \mathbf{v}_{3}-L_{55} \mathbf{u}_{5}-L_{56} \mathbf{u}_{6}-$ $L_{53} \mathbf{u}_{3}+L_{51} \mathbf{u}_{1}$. Each equation has

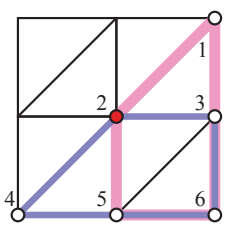
exactly one variable $\mathbf{u}_{2}$; the remaining components of $\mathbf{u}$ and $\mathbf{v}$ are on the boundary and are fixed. In general, these equations are not compatible. Similar observation holds for the full mass matrix, but a slightly larger neighborhood needs to be considered.

Reduction of fixed curve boundary conditions. An alternative approach to discretizing curve boundary conditions is to reduce them to the discretization of the type (13). This is achieved by inferring the values of $\mathbf{u}_{i}$ for $i \in I_{-1}$ where $I_{-1}$ are the vertices in the interior of $\Omega$, edge-adjacent to the boundary, from the boundary values of $\partial \mathbf{u} / \partial n=\mathbf{b}_{1}$.

For each triangle in $\Omega$ with 2 vertices on the boundary, the value of the interior vertex can be easily determined independently, as the $\partial \mathbf{u} / \partial n$ and the boundary values completely specify the gradient of $\mathbf{u}$. If several such triangles have a common vertex $v$ in the interior (the situation that leads to overdetermined systems), we simply average all values obtained for $v$ from these triangles, since the difference in these values should decrease at least as $O\left(h^{2}\right)$ as the mesh is refined. Algebraically, this procedure eliminates a part of the overdetermined system by exploiting the fact that the values of $\mathbf{u}$ on $I_{-1}$ can be solved for in least-squares sense, independently of the rest of the variables.

As a result, we obtain a discrete system of equations with two rows of values of $\mathbf{u}$ fixed, and values of $\mathbf{v}$ on the boundary. The rest of the derivation proceeds as before, by substituting piecewise linear expressions for $\mathbf{u}$ (with two rows fixed), $\mathbf{v}$ (with one row fixed) and $\lambda$ (with no rows fixed). The system matrix is identical to (13), if one row on the boundary is removed from $\Omega$, and renamed $I_{0}$ and $I_{0}$ is renamed $I_{1}$. The right-hand side has the same form, but instead of $\mathbf{v}_{1}$ given by (14), the boundary value of $\mathbf{v}_{1}$ appears directly.

Convergence. While many versions of theoretical analysis are available for the biharmonic and other fourth-order problems, much less work was done on higher-order equations. Unfortunately, the triharmonic problem suffers from the same diffuculty as biharmonic: general theorems based on the LBB (inf-sup) condition do not apply for similar reasons. Mixed elements for polyharmonic problems are considered in [BF85]; however, error estimates are obtained under the assumption that the discretization in the domain $\Omega$ is asymptotically finer than the discretization on the boundary.

\section{Evaluation and Applications}

Implementation. In our implementation, UMFPACK is used to solve the system in all cases (note that a Cholesky solver cannot be used unless the system is reduced to a single 

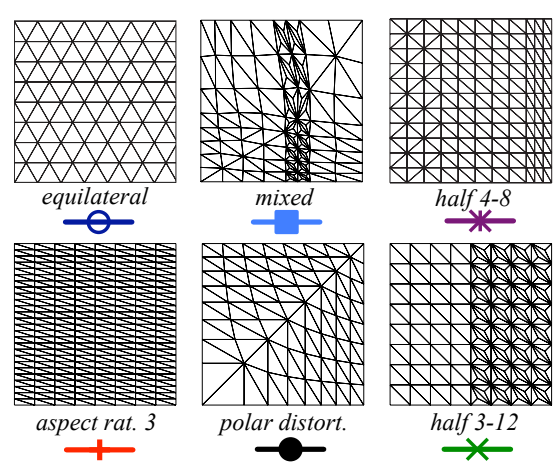

Figure 6: Mesh connectivities used in tests, from [GGRZ06].

variable). We observe that the timings for solving the system with auxiliary variables do not differ much from timings for solving the reduced system: while the number of variables is smaller, the system is much less sparse. As only the righthand side depends on the boundary conditions, the system can be prefactored when these conditions are manipulated.

Convergence and mesh dependence. We start with a study of the errors of the region and curve boundary conditions. We characterize meshes by the average edge length $h$. We have used several sequences of meshes from [GGRZ06]. We also used several sets of randomized meshes, obtained by perturbing the positions of points on a regular grid and running Delaunay triangulation on the resulting vertices, with Steiner points added to satisfy a constraint on the minimal angle. The errors are calculated by comparing our solution with an analytic solution. We choose an arbitrary function $u^{t}$ as the target analytic solution, sample boundary conditions from this function and use the right-hand side $g=\Delta^{k} u^{t}$ for $k=2$ and $k=3$. We have tried a number of test functions with similar results.

Figs. 7 and 8 show how the $L^{2}$ error changes with average edge length for randomized meshes with minimal angle of 30 and 1 degrees, and Fig. 9 shows the same dependence for the meshes from Fig. 6. We observe that the convergence rate for the Laplacian energy (biharmonic equation) is consistent with the error estimate of [Sch78]. There is no significant difference between the behaviors of region and curve boundary condition formulations, and there is good consistency between different connectivities with the same average edge length. At the same time, more regular mesh patterns result in greater dependence on connectivity (Fig. 9, left). There is no significant difference in numerical solutions obtained from the reduced formulation (11) and the equivalent two-variable formulations (10) and (12).

The behavior for the Laplacian gradient is quite different (Figs. 8 and 9, right). The observed average convergence rate is substantially slower for region conditions, and the deviation from the average error for a given mesh resolution is higher. The most significant observed effect is that by using curve conditions with explicitly defined second derivative, we obtain substantially better results, both in terms of error magnitude and lesser mesh dependence. Reduced single-
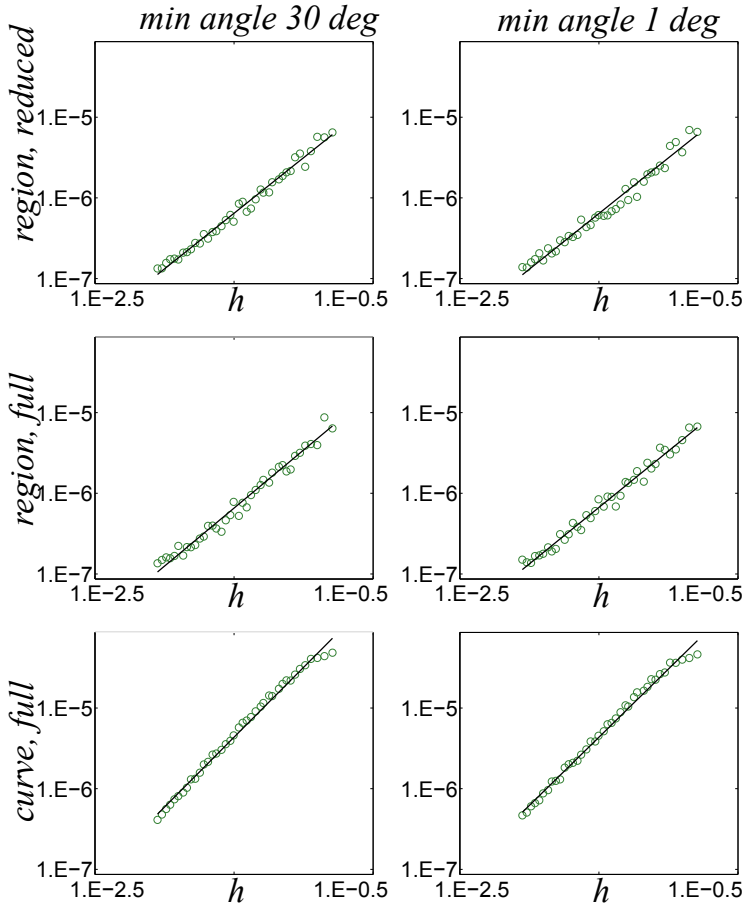

Figure 7: Dependence of the error on average edge length for the Laplacian energy, randomized meshes.

variable systems of the type (11) produce stronger mesh dependence and in some cases inferior convergence behavior compared to the full mixed element system.

Mass matrix lumping. While the convergence rate and mesh dependence are not strongly affected by the choice of the mass matrix lumping strategy, there is a substantial difference in error magnitudes, which is consistent with the observations in the literature. Fig. 10 shows a comparison of "barycentric" lumping, with each diagonal entry obtained as $1 / 3$ of the sum of areas of triangles incident at the vertex, and the "hybrid" approach, using Voronoi areas for non-obtuse triangles [MDSB02].

Applications. Figs. 2, 3 show examples of interactive editing of biharmonic and triharmonic surfaces, with different user-defined boundary conditions. The user is free to manipulate single points, curves or regions in the edited shape. Tangents can be prescribed along curve boundaries for biharmonic surfaces; triharmonic surfaces additionally admit second derivative control along the direction perpendicular to the boundary. Tangents are explicitly specified by the user at a sparse set of points along the boundary curve using a simple vector widget, and the tangents at the remaining points are computed by interpolation (Fig. 4). Second derivatives are set using the Bézier widget (Figs. 3, 15): the control triangle of a quadratic Bézier curve whose derivatives are used in the boundary conditions for $\partial / \partial n$ and $\partial^{2} / \partial n^{2}$.

Smooth detail-preserving deformations (Fig. 15) are achieved by solving the bi- or triharmonic equation for the displacement function $\mathbf{u}$. The user can interactively manip- 

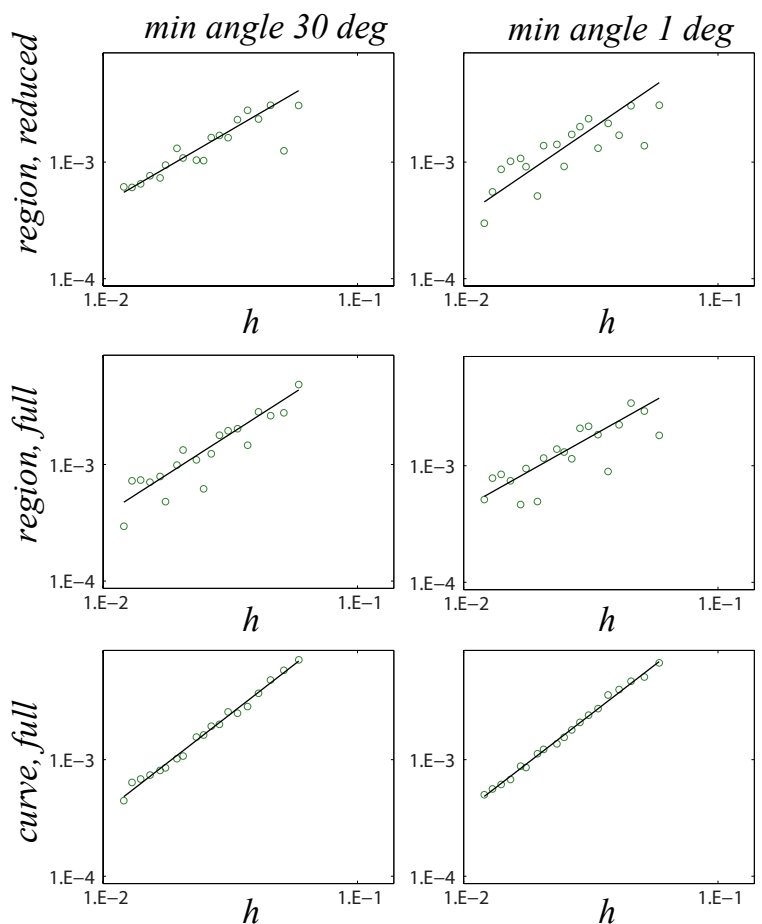

Figure 8: Dependence of the error on average edge length for the Laplacian gradient energy, randomized meshes.

ulate the boundary conditions for the displacement field, namely positions, first and second derivatives. A more complex approach involving rotations [BS08] can also be formulated in the mixed-element framework.

Another application of PDE-based surfaces is smooth hole filling and blending between shapes. In hole filling, a surface with a boundary loop (a hole) is given, whereas blending implies two or more loops that need to be connected by a surface. One can use the region constraints to ensure $C^{1}$ or $C^{2}$ continuity with the rest of the surface, or curve boundary constraints to specify tangents and/or curvatures at the hole-border directly (Fig. 11). Blending between two shapes using region boundary conditions is shown in Fig. 12; the higher smoothness of the triharmonic surface is evident from the reflection lines. Fig. 14 shows two curves interpolated with a variational patch, with the shape of the patch controlled by tangents and second derivatives specified along the curves (we map both curves to two opposite boundaries of a rectangular area in the plane, and periodic conditions are imposed at the other two boundaries).

\section{Conclusions}

The technique that we have presented allows one to discretize a broad variety of functionals and PDEs with different types of boundary conditions using only piecewise linear elements. The main components of the approach include (1) factorization of the original equations into low-order equations by introducing additional variables; (2) using constraints to impose region conditions; (3) lumping mass matrices to eliminate unneeded variables.
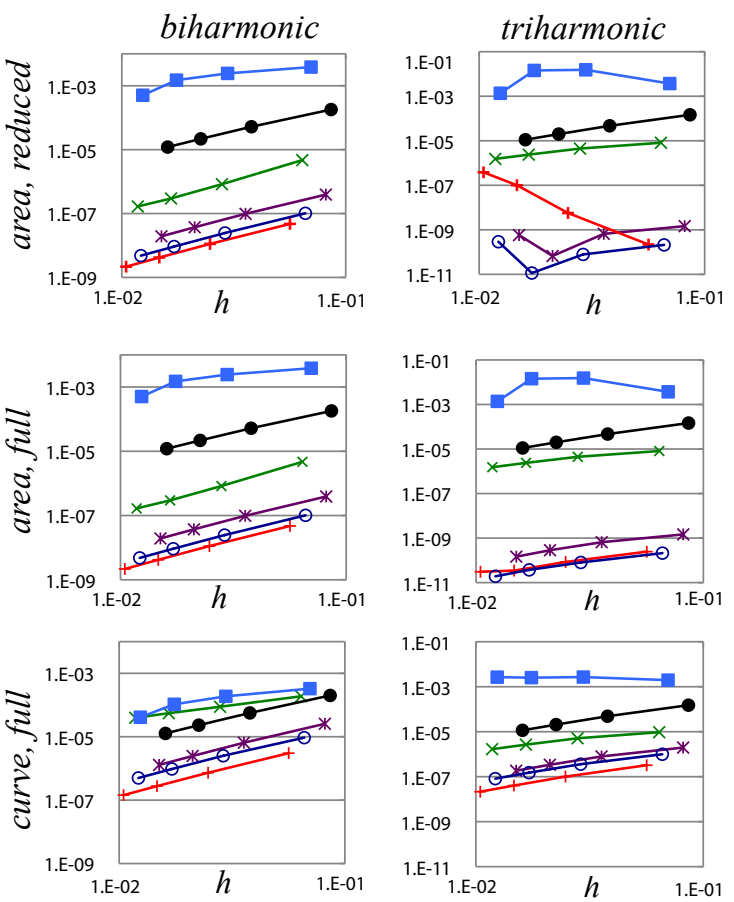

Figure 9: Dependence of the error on average edge length for the Laplacian energy (left), and Laplacian gradient energy (right); test mesh connectivities shown in Fig. 6.

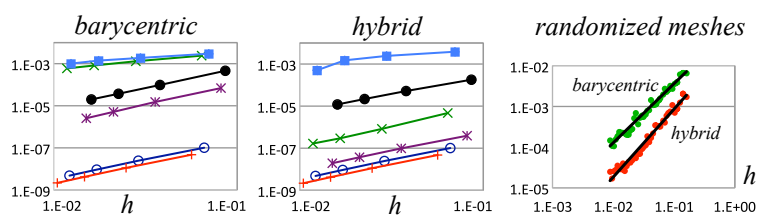

Figure 10: Comparison of barycentric and hybrid [MDSB02] lumped mass matrices.

While experimental evidence shows that the method is converging for both forth- and sixth- order problems, convergence is significantly slower for the latter, and mesh dependence is stronger. We observe that using high-order interpolation to estimate the second-order boundary condition somewhat improves the situation.

One potential direction for improvement is, instead of factoring this system into three second-order systems, to use a fourth- and a second-order system, with quadratic elements for the former (e.g., the ones used in [GGRZ06]).

In the simplest case (biharmonic equation) existing theory can be used to establish convergence guarantees; much less is known for sixth-order systems, and the experimentally observed convergence rates are significantly lower. Another important direction for exploration is the effect of the non-flat metric, which we did not consider in this paper; the discretizations we describe should still apply with no significant changes. 


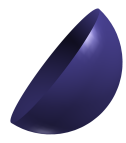

original

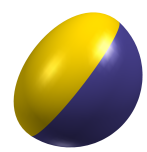

$\Delta^{2}$, fixed region

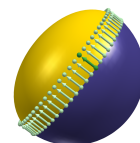

(1)

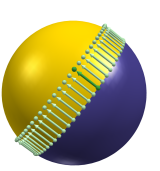

$\Delta^{2}$, fixed tangents
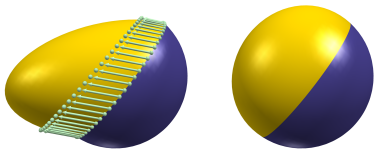

$\Delta^{3}$, fixed region $\Delta^{3}$, fixed $\partial^{2} / \partial n^{2}$

Figure 11: Hole filling: the half-sphere mesh was completed to close the "hole". The left images show biharmonic reconstruction using region constraints and curve constraints with different prescribed tangents. The two rightmost images show the triharmonic reconstruction using region constraints and curve constraints with user-prescribed curvatures.

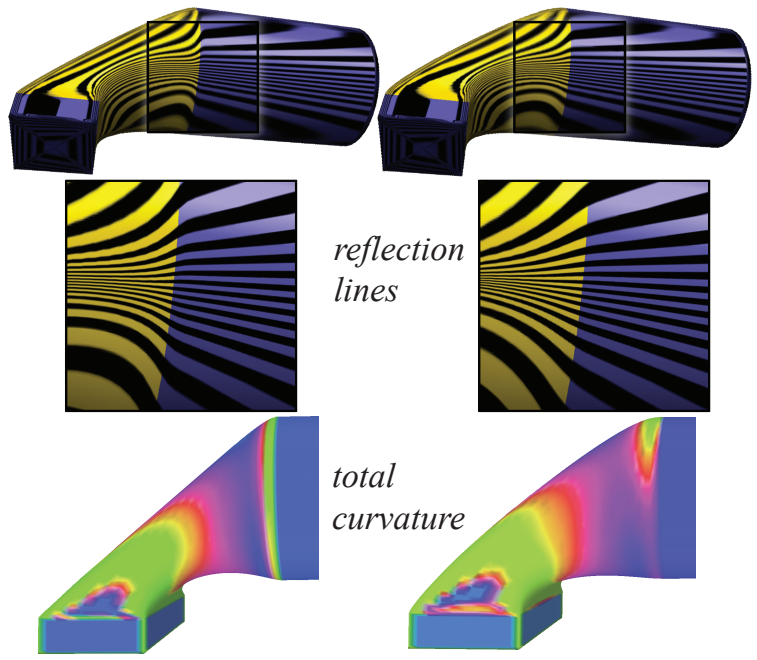

Figure 12: Blending between surfaces (cylinders with a square and circular cross-sections) using bi- and triharmonic equations and region boundary conditions. Note the smoother behavior of reflection lines in the triharmonic case.

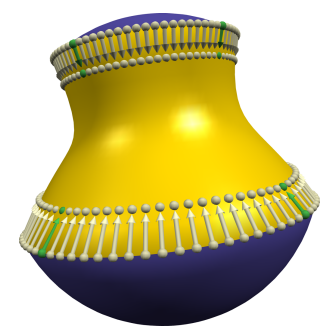

Figure 13: Blending between two spherical caps, with controllable sharp features introduced using tangent conditions.

\section{Acknowledgment}

This work was supported in part by an NSF award IIS0905502.

Appendix A: Ciarlet-Raviart discretization and region boundary conditions.

We outline the connection between solutions to the systems (12) and (10) here; a full rigorous treatment would require detailing assumptions on the smoothness spaces for boundary data and is beyond the scope of this paper. To simplify

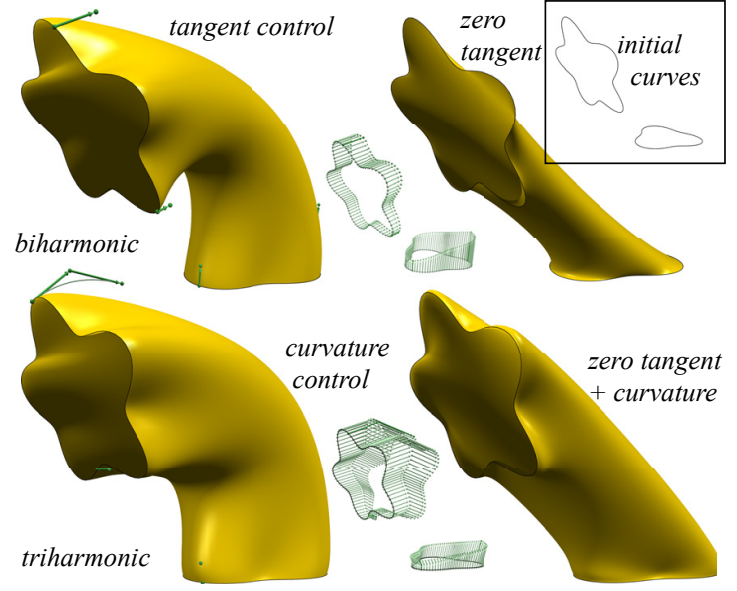

Figure 14: Filling in a patch between surfaces. Tangents or tangents + second derivatives can be specified at curves to obtain the desired shape.

consideration, we assume that the solutions are classical solutions, i.e., $\mathbf{u}$ is four times differentiable in $\Omega$. In this case, solutions of continuous problems with curve boundary conditions and region boundary conditions are identical, as long as the boundary conditions for the curve problem are sampled from $\mathbf{u}^{f}$ for the region problem.

We consider a simplified situation with homogeneous Dirichlet conditions, i.e., we assume that for (10), $\mathbf{u}^{f}=0$, and for (12), $\mathbf{b}_{0}=0$ (this is a standard reduction for Dirichlet conditions, using substitution $\mathbf{u}=\mathbf{u}^{\text {orig }}-\mathbf{u}^{D}$ where $\mathbf{u}^{D}$ satisfies the Dirichlet condition [Bra02]). This reduction requires introducing a right-hand side for the second equation in the system: $\Delta \mathbf{u}=\mathbf{v}, \Delta \mathbf{v}=\Delta^{2} \mathbf{u}^{D}=\mathbf{g}$.

The Ciarlet-Raviart system (12) with lumped mass matrix with solution $\left(\mathbf{v}_{\bar{\Omega}}^{*}, \mathbf{u}_{\Omega}^{*}\right)$ can be rewritten in the form

$$
\left[\begin{array}{cc}
-M^{d} & L_{\bar{\Omega}, \Omega} \\
L_{\Omega, \bar{\Omega}} & 0
\end{array}\right]\left[\begin{array}{l}
\mathbf{v}_{\bar{\Omega}} \\
\mathbf{u}_{\Omega}
\end{array}\right]=\left[\begin{array}{c}
-N_{\bar{\Omega}, 0}^{\partial \Omega} \mathbf{b}_{1}-M_{\bar{\Omega}, 0}^{d, \Omega^{e}} \mathbf{v}_{0}^{*} \\
\mathbf{g}^{\Omega}
\end{array}\right],
$$

where we have subtracted $M_{\bar{\Omega}, 0}^{d, \Omega^{e}} \mathbf{v}_{0}^{*}$ from both sides, to obtain the same left-hand side as in (10), and $\mathbf{g}_{j}^{\Omega}=\left\langle\mathbf{g}, \varphi_{j}\right\rangle_{\Omega}$. In comparison, the right-hand side of (10) is $\left[0, \mathbf{g}^{\Omega_{0}}\right]$, with $\mathbf{g}_{j}^{\Omega_{0}}=\left\langle\mathbf{g}, \varphi_{j}\right\rangle_{\Omega_{0}}$. As shown in [Sch78], $\left(\mathbf{v}^{*}\right)^{h}$ converges to $\mathbf{v}$ in in $L^{2}$-norm, and $\mathbf{v}$ is at least continuous on $\bar{\Omega}$. It can be extended by zero to all of $\Omega_{0}$, consistently with 


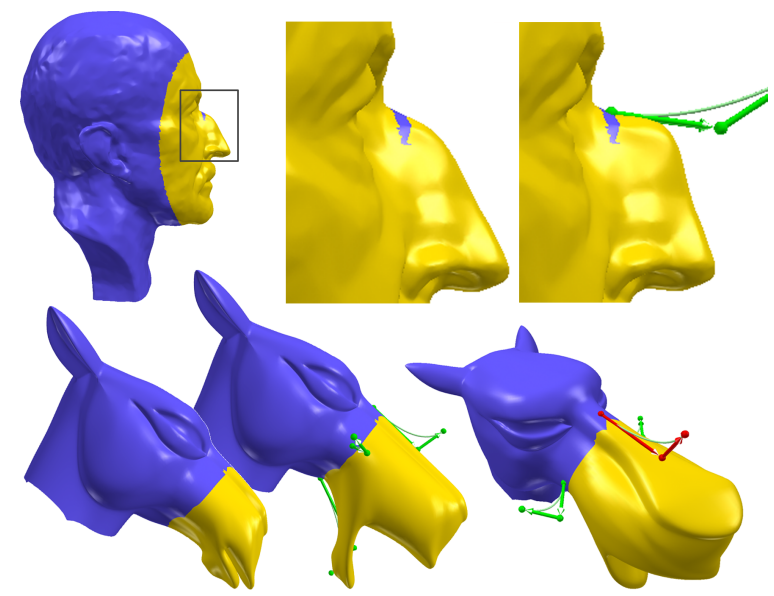

Figure 15: Controlling deformations using tangents and second derivatives. We solve the triharmonic equation $\Delta^{3} \mathbf{u}=0$ for the displacement function (the edited surface is then $\mathbf{x}+\mathbf{u})$. The Camel's nose is lengthened and the mouth opened by manipulating both the first and the second normal derivatives of the displacement field; the curvature of the Max Planck's nose is altered by interacting with the second derivatives via the Bézier widget.

$\Delta \mathbf{u}^{f}$. On the other hand, the $j$-th component of $-N_{\bar{\Omega}, 0}^{\partial \Omega} \mathbf{b}_{1}$, $-\left\langle\mathbf{b}_{1}, \varphi_{j}\right\rangle_{\partial \Omega}=\left\langle\nabla u^{f}, \nabla \varphi_{j}\right\rangle_{\Omega_{e}}=-\left\langle\mathbf{b}, \varphi_{j}\right\rangle_{\partial \Omega}=\left\langle\mathbf{v}, \varphi_{j}\right\rangle_{\Omega_{e}}$. Combining $L^{2}$ convergence of solutions $\left(\mathbf{v}^{*}\right)^{h}$ of (10) to $\mathbf{v}$, and the fact that components of $M_{\bar{\Omega}, 0}^{d, \Omega^{e}} \mathbf{v}_{0}^{*}$ are quadrature approximations of $\left\langle\mathbf{v}^{*}, \varphi_{j}\right\rangle_{\Omega_{e}}$, we observe that the r.h.s. of (16) converge in $L^{2}$ norm to the the r.h.s. of the system obtained for the (12) formulation in $L^{2}$ norm, so the difference in the solutions also converges as shown in [BF91].

\section{References}

[AD01] AmARA M., DABAGHI F.: An optimal $C^{0}$ finite element algorithm for the 2D biharmonic problem: theoretical analysis and numerical results. Numer. Math. 90, 1 (2001), 19-46.

[BF85] BRAmble J., FALK R.: A mixed-Lagrange multiplier finite element method for the polyharmonic equation. Mathematical Modelling and Numerical Analysis 19, 4 (1985), 519-557.

[BF91] BREZZI F., ForTin M.: Mixed and hybrid finite element methods, volume 15 of Springer Series in Computational Mathematics. Springer-Verlag, New York 2, 5 (1991), 2.

[BK04] Bотsch M., Kobвelt L.: An intuitive framework for real-time freeform modeling. ACM Trans. Graph. 23, 3 (2004), 630-634.

[Bra02] BRAESS D.: Finite Elements: Theory, fast solvers and applications in solid mechanics, 2nd edn, vol. 13. 2002.

[BS08] BOTSCH M., SORKINE O.: On linear variational surface deformation methods. IEEE Transactions on Visualization and Computer Graphics 14, 1 (2008), 213-230.

[BW90] BLOOR M. I. G., WILsON M. J.: Using partial differential equations to generate free-form surfaces. Computer Aided Design 22, 4 (1990), 202-212.

[CDD*04] Clarenz U., Diewald U., Dziuk G., RumpF M., RUSU R.: A finite element method for surface restoration with smooth boundary conditions. Comput. Aided Geom. Design 21, 5 (2004), 427-445.
[CG91] Celniker G., Gossard D.: Deformable curve and surface finite-elements for free-form shape design. In Proceedings of ACM SIGGRAPH (1991), pp. 257-266.

[Cia78] CIARLET P.: The finite element method for elliptic problems. North-Holland, 1978.

[CR74] Ciarlet P. G., RaViart P.-A.: A mixed finite element method for the biharmonic equation. In Mathematical aspects of finite elements in partial differential equations (Proc. Sympos., Math. Res. Center, Univ. Wisconsin, Madison, Wis., 1974). Math. Res. Center, Univ. of Wisconsin-Madison, Academic Press, New York, 1974, pp. 125-145. Publication No. 33.

[DD06] DECKELNiCK K., DzIUK G.: Error analysis of a finite element method for the Willmore flow of graphs. Interfaces and free boundaries 8,1 (2006), 21.

[Dzi88] DzIUK G.: Finite elements for the Beltrami operator on arbitrary surfaces. Partial differential equations and calculus of variations (1988), 142-155.

[Dzi90] DzIUK G.: An algorithm for evolutionary surfaces. $\mathrm{Nu}$ merische Mathematik 58, 1 (1990), 603-611.

[Fal78] FALK R.: Approximation of the biharmonic equation by a mixed finite element method. SIAM Journal on Numerical Analysis 15, 3 (1978), 556-567.

[GGRZ06] GRINSPUN E., Gingold Y., REISMAN J., ZORIN D.: Computing discrete shape operators on general meshes. In Computer Graphics Forum (2006), vol. 25, pp. 547-556.

[MDSB02] MEYer M., Desbrun M., SChröDER P., BARR A.: Discrete differential-geometry operators for triangulated 2manifolds. Visualization and mathematics 3 (2002), 35-57.

[Mon87] Monk P.: A mixed finite element method for the biharmonic equation. SIAM Journal on Numerical Analysis 24, 4 (1987), 737-749.

[MS92] Moreton H. P., SÉQuin C. H.: Functional optimization for fair surface design. In Proceedings of ACM SIGGRAPH (1992), pp. 167-176.

[NISA07] Nealen A., Igarashi T., Sorkine O., Alexa M.: FiberMesh: designing freeform surfaces with $3 \mathrm{D}$ curves. $A C M$ Trans. Graph. 26, 3 (2007), 41.

[PP93] Pinkall U., Polthier K.: Computing discrete minimal surfaces and their conjugates. Experiment. Math. 2, 1 (1993), 1536.

[RBG*09] Reuter M., Biasotti S., Giorgi D., PATANČ G., SPAGNUOLO M.: Discrete Laplace-Beltrami operators for shape analysis and segmentation. Computers \& Graphics 33, 3 (2009), 381-390.

[Sch78] Scholz R.: A mixed method for 4th order problems using linear finite elements. RAIRO Anal. Numér. 12, 1 (1978), 85-90, iii.

[SK00] SchneIdeR R., Kobbelt L.: Generating fair meshes with $G^{1}$ boundary conditions. In Geometric Modeling and Processing Conference Proceedings (2000).

[SK01] SCHNEIDER R., KobBELT L.: Geometric fairing of irregular meshes for free-form surface design. Computer Aided Geometric Design 18, 4 (May 2001), 359-379.

[Tau95] TAUBIN G.: A signal processing approach to fair surface design. In Proceedings of ACM SIGGRAPH (1995), pp. 351358.

[WBH*07] WARDETZKy M., Bergou M., Harmon D., ZORIN D., GRINSPUN E.: Discrete quadratic curvature energies. Computer Aided Geometric Design 24, 8-9 (2007), 499-518.

[WW94] WELCH W., WITKIN A.: Free-form shape design using triangulated surfaces. In Proceedings of ACM SIGGRAPH (1994), pp. 247-256.

[XPB06] XU G., PAN Q., BAJAJ C. L.: Discrete surface modelling using partial differential equations. Comput. Aided Geom. Design 23, 2 (2006), 125-145. 